\title{
Previsões para a produção de leite sob instabilidade pluviométrica no Ceará no período de 1974 a 2019
}

\author{
Forecasts for milk production under rainfall instability in Ceará on \\ the period of 1974 to 2019. \\ Elizama Cavalcante de Paiva' (1), José de Jesus Sousa Lemos $^{1}$ (D), Robério Telmo Campos ${ }^{1}$ (1) \\ ${ }^{1}$ Programa de Pós-graduação em Economia Rural, Universidade Federal do Ceará (UFC), Fortaleza (CE), Brasil. E-mails: \\ elizamapaiva@yahoo.com.br; lemos@ufc.br; roberio@ufc.br
}

\begin{abstract}
Como citar: Paiva, E. C., Lemos, J. J. S., \& Campos, R. T. (2022). Previsões para a produção de leite sob instabilidade pluviométrica no Ceará no período de 1974 a 2019. Revista de Economia e Sociologia Rural, 60(spe), e252091. https:// doi.org/10.1590/1806-9479.2021.252091
\end{abstract}

Resumo: O setor da pecuária é fortemente influenciado pelos fenômenos climáticos, e a instabilidade pluviométrica se torna um entrave à capacidade produtiva, sobretudo dos municípios pertencentes ao semiárido. Os produtores planejam e organizam sua produção a partir de experiências passadas e criam expectativas quanto ao futuro. Este trabalho avaliou a forma como as variáveis exógenas (pluviometria e preços) interferiam nas previsões dos produtores de leite no Ceará, no período de 1974 a 2019. Geraramse projeções acerca das variáveis endógenas sobre as quais os produtores de leite tinham poder de decisão (rebanho e produtividade). Embora eles não tivessem poder de decisão sobre o preço médio, que é determinado exogenamente pelo mercado, o estudo fez estimativas das formas de projeções também para essa variável. Estimou-se a maneira como provavelmente a pluviometria pudesse afetar as previsões das variáveis associadas à produção de leite. Utilizou-se do método ARIMA, proposto por Box e Jenkins (1976), para captar o comportamento das variáveis com base em séries históricas (1974-2019). Os resultados confirmaram o impacto indireto das chuvas e dos preços sobre as variáveis endógenas de decisão. As trajetórias das expectativas de produção e dos valores projetados, bem como os testes estatísticos realizados, sinalizaram a robustez dos ajustamentos feitos na pesquisa.

Palavras-chave: semiárido, instabilidade pluviométrica, projeções, tecnologias.

\begin{abstract}
The livestock sector is strongly influenced by weather phenomena and rainfall instability becomes an obstacle to productive capacity, especially in municipalities belonging to the semiarid region. Producers plan and organize their production based on past experiences, and from these experiences, they create their expectations for the future. This work evaluated how exogenous variables (rainfall and prices) interfere in the forecasts of dairy farmers in Ceará in the period from 1974 to 2019. Projections are generated about the endogenous variables over which dairy farmers have decision-making power (herd and productivity). Although they do not have decision-making power over the average price, which is exogenously determined by the market, the study estimated the forms of projections for this variable as well. It was also estimated how rainfall likely affects the predictions of variables associated with milk production. The ARIMA method proposed by Box and Jenkins (1976) was used to capture the behavior of variables based on their historical series (1974-2019). The results confirmed the indirect impact of rainfall and prices on the endogenous decision variables. The trajectories of production expectations and projected values, as well as the statistical tests performed, indicated the robustness of the adjustments made in the survey.
\end{abstract}

Keywords: semiarid, pluviometric instability, projections, technologies

\section{Introdução}

O estado do Ceará ocupa uma extensão territorial de $148.016 \mathrm{~km}^{2}$, incluindo a superfície hídrica, e possui 184 municípios distribuídos em 33 microrregiões que compõem as sete mesorregiões. O seu território está quase todo inserido em regiões de climas semiáridos, e sua economia é intensivamente influenciada pelos fenômenos climáticos e pela instabilidade 
no regime pluviométrico, que tem como uma das características importantes ser cíclica na escassez de chuvas e de apresentar má distribuição espacial e temporal. Esses aspectos da instabilidade pluviométrica se manifestam como importantes definidores da produção do Estado, afetando o rendimento das culturas, os níveis de produção, as expectativas e o planejamento dos produtores rurais quanto às suas produções futuras.

Segundo Lemos (2020), os municípios do semiárido apresentam, além de vulnerabilidades naturais associadas aos problemas climáticos, indicadores de vulnerabilidades induzidas, que penalizam as populações dos municípios ali situados de forma maior do que nos municípios que estão fora dessa localidade. Assim, as vulnerabilidades naturais provocadas por escassez hídrica cíclica e as induzidas são fortes indutoras dos padrões elevados de pobreza observados no Nordeste em geral e, em especial, no semiárido dessa região.

Das exposições anteriores, depreende-se que os locais inseridos no semiárido que têm grande parte de sua economia baseada na agricultura ou pecuária de sequeiro são diretamente afetados por irregularidades climáticas que dificultam e até inviabilizam o planejamento dos negócios, quer seja de base familiar, quer seja nas unidades agrícolas não familiares.

A instabilidade climática, que afeta todos os estados do Nordeste, impacta de forma significativa a pecuária regional, porque reduz a oferta e a qualidade da pastagem e causa e incrementa a sazonalidade da oferta de leite ao longo dos anos (Ximenes, 2009).

Reis Filho \& Carvalho (2009) concordam com essa assertiva de Ximenes (2009) e acrescentam que são vários os fatores que resultam em baixa produtividade da produção leiteira no Nordeste, entre os quais citam: a sazonalidade da oferta de forragem, o baixo nível tecnológico e a necessidade de fortalecer e modernizar o parque industrial. Contudo, a baixa e, principalmente, a irregular pluviosidade sempre foram, e continuarão sendo, os grandes obstáculos a serem superados.

Dentro desse cenário, encontra-se a figura do sertanejo cearense que busca conviver com as imposições de um ambiente hostil, criando estratégias de sobrevivência apoiadas em conhecimentos empíricos, que possibilitem minimizar os riscos de perda da sua produção. Entre esses conhecimentos, Folhes \& Donald (2007) citam os respeitados "profetas das chuvas", que se baseiam em observações tradicionais e empíricas do ambiente para interpretar a instabilidade climática da região e as suas implicações diretas na produção agrícola e pecuária. Fazem previsões para que os agricultores cearenses, com o amparo nelas, tomem providências de modo a minimizar as vulnerabilidades naturais (instabilidade pluviométrica) a que estão expostos.

O fato é que os produtores rurais, em geral, e os de leite, em particular, detêm um acervo de conhecimentos e de informações, boa parte delas decorrente da sua própria trajetória, de familiares e amigos. Assim, buscam projeções acerca dos preços que receberão ao produzirem o leite ao longo da sua trajetória, apesar de a variável ser exógena e totalmente fora da sua capacidade de interferência.

Contudo, trabalha-se neste estudo com a suposição de que os preços do leite também afetam as iniciativas de planejamento acerca do tamanho do rebanho e do padrão tecnológico a ser utilizado, que interferirá na produtividade das vacas em lactação. Outra variável exógena que afeta a decisão de ampliar ou reduzir o tamanho do rebanho e a tecnologia a ser utilizada é a pluviometria, de grande relevância em um estado que tem praticamente o seu território todo inserido no semiárido. Como as informações se acumulam ao longo dos anos, supõese que, com o passar dos anos, os agricultores consigam ser mais rigorosos na formação de expectativas e, portanto, ter mais chances de errar menos. 
Assim, nesta pesquisa, busca-se avaliar a forma como as variáveis exógenas (pluviometria e preços) interferem nas previsões dos produtores de leite no Ceará no período de 1974 a 2019.

De forma específica, o estudo busca: a) mostrar as estatísticas descritivas das variáveis envolvidas na produção de leite no Ceará entre 1974 e 2019, destacando o coeficiente de variação de cada uma delas; b) estimar como os produtores de leite no semiárido cearense elaboraram as suas projeções acerca do tamanho do rebanho, da produção diária de leite e dos preços de leite no semiárido do Ceará no período de 1974 a 2019; c) aferir as formas como o choque exógeno provocado pela imprevisibilidade da precipitação de chuvas e dos preços pode ter afetado as previsões do tamanho do rebanho e da produção diária de leite no Ceará no período investigado.

\section{Fundamentação teórica: semiárido no Ceará}

No Nordeste semiárido, a ocorrência de instabilidade climática é uma variável exógena sempre presente, mas de difícil previsão acerca da magnitude dos seus efeitos. Isso dificulta a formação de expectativa das diferentes atividades econômicas que se planejam para a região, sobretudo para os territórios inseridos nesse ecossistema (Fama, 1969).

O semiárido do Nordeste brasileiro é constituído por várias sub-regiões, onde predomina uma diversificação de clima, vegetação, solo, água e aspectos socioeconômicos (Silva et al., 1993). Contudo, quando há períodos prolongados de estiagem, praticamente toda a região sofre com as calamidades da seca, independentemente de suas diversidades geoambientais. Nela, a agricultura, sobretudo a produtora de alimentos, praticada em sua maioria por agricultores familiares, e a pecuária extensiva são de alto risco, visto que as produtividades médias alcançadas pelas principais culturas alimentares cultivadas ficam muito abaixo dos valores obtidos em outras regiões. A produção e a produtividade do leite também são afetadas por essas instabilidades na precipitação de chuvas.

De acordo com a última revisão do mapa do semiárido, realizado pelo Conselho Deliberativo da Superintendência do Desenvolvimento do Nordeste (CONDEL/SUDENE), em reunião realizada em 23 de novembro de 2017, politicamente o semiárido brasileiro passou a contar com 1.262 municípios, 129 a mais do que o montante que fazia parte desse regime climático definido na última atualização feita em 2005 por essa instituição. Na nova delimitação, o Ceará, que antes possuía 150 municípios reconhecidos oficialmente como incluídos no semiárido brasileiro, passou a ter 175 dos seus 184 municípios (95\%) reconhecidos como fazendo parte desse ecossistema (Brasil, 2017).

Com a nova delimitação oficial que apenas reconhece uma situação climática que já prevalecia no estado, a despeito do reconhecimento oficial do Governo Federal, depreende-se que o Ceará é, proporcionalmente, o estado brasileiro com a maior inserção de municípios no semiárido brasileiro e com a maior população diretamente inserida nesse ecossistema.

Em razão do fato de as atividades agrícolas dependerem bastante dos fatores climáticos, sobretudo da pluviometria, elas se tornam mais sensíveis às instabilidades do clima, dado que, mesmo com todos os avanços tecnológicos, dispor de condições climáticas favoráveis ainda é considerado um fator-chave para a obtenção de bons níveis de produtividade agrícola, o que mostra a alta vulnerabilidade do setor agrícola diante da instabilidade climática que permeia a realidade da população rural, que tem como principal atividade econômica a agricultura (Deschênes \& Greenstone, 2007; Ximenes, 2013; Lemos \& Bezerra, 2019).

A produção de leite no Nordeste está entre as atividades que dependem das oscilações climáticas, especialmente na região semiárida, onde se processa praticamente toda a produção 
de leite do Ceará e, assim, contribui para um baixo desempenho no setor leiteiro, fazendo com que os produtores situados nessa região apresentem algumas das menores produções diárias de leite por vaca ordenhada do Brasil (Instituto Brasileiro de Geografia e Estatística, 2017).

A Pesquisa Pecuária Municipal (PPM) é uma das principais fontes de informações sobre a pecuária e passou a ser gerida pelo Instituto Brasileiro de Geografia e Estatística (IBGE) a partir de 1974, de acordo com o Decreto n 73.482, de 17 de janeiro de 1974 - eis o motivo de o presente estudo abordar esse espaço temporal a partir desse ano. Ainda de acordo com a PPM (Instituto Brasileiro de Geografia e Estatística, 2019), a produtividade de leite no Brasil foi de 2,14 litros diários por vaca, enquanto no Ceará a produtividade diária foi de 1,37 litro/ vaca. Segundo Thornton et al. (2009), essa diferença se justifica, sobretudo, por causa das raças e genéticas do rebanho leiteiro, das técnicas no manejo dos animais, na ordenha, no controle de doenças, na qualidade da mão de obra, como também das instalações em que esses animais são mantidos. Ximenes (2009) e Galvão Júnior et al. (2015) concordam com as assertivas de Thornton, mas acrescentam que essa baixa produtividade está diretamente ligada ao contexto pluviométrico da região que compromete a produção de leite, bem como afeta a produção de forragens, o agravamento e surgimento de doenças e mais, especialmente, a disponibilidade de água.

Mesmo com todas essas dificuldades, a pecuária, conforme Paiva (2018) afirma, cumpre um importante papel no desenvolvimento socioeconômico do estado, gerando trabalho e renda para milhares de cearenses que vivem nas áreas rurais do estado. Informações do Censo Agropecuário 2017 enfatizam a afirmativa que, dos 394.330 estabelecimentos agropecuários, 73 mil trabalham na produção de leite (Instituto Brasileiro de Geografia e Estatística, 2017). Ao comparar com o Censo de 2006, observa-se uma redução do número de estabelecimentos em 12\%, entretanto a produção aumentou em mais de 30\%, passando de 459.331 mil litros de leite por ano (Instituto Brasileiro de Geografia e Estatística, 2006) para 606.764 mil litros por ano (Instituto Brasileiro de Geografia e Estatística, 2017). Esse indicador exibe uma melhora na qualidade do rebanho leiteiro cearense.

A disponibilidade de água é um fator essencial e decisivo para a produção de leite. Assim, fazer avaliações constantes a respeito da disponibilidade hídrica se torna uma ação decisiva e necessária a esses produtores. A Instrução Normativa $n^{\circ} 62$ determina que a propriedade deva garantir 100 litros de água por animal a ser ordenhado, além de 6 litros de água para cada litro de leite produzido (Brasil, 2011). Logo, a água é um item essencial para a produção de leite. De acordo com Gonçalves et al. (2015), a água entra com 87\% na composição de cada litro de leite produzido. Sendo assim, a disponibilidade de água de qualidade à vontade para as vacas em ordenha é fator decisivo, assim como ração e pastejo adequados, para os criadores auferirem maiores produtividades físicas e maiores retornos econômicos por animal em lactação (Gonçalves et al., 2015).

\section{Metodologia}

A base de dados utilizada neste trabalho foi construída a partir de informações coletadas na PPM, disponibilizada pelo IBGE. O período de análise se estende de 1974 a 2019.

\section{Formulação teórica do trabalho}

Neste estudo, assumiu-se que os produtores de leite estabeleciam planejamentos e previsões nas suas futuras produções de leite com base em informações disponíveis no passado, provendo 
um maior destaque (ponderação) para as informações mais recentes. Assim, o modelo teórico tentou captar o papel exercido pelos choques ocorridos na produção de leite. Com base nessas informações, a pesquisa supôs que os agentes dispunham de escolhas para auxiliar na tomada de decisão sobre o que e quanto produzir, dadas as informações que reuniam para fazer as suas antevisões.

O estudo utilizou as seguintes variáveis a partir das quais se supôs que os agricultores tomassem as suas decisões para a produção de leite no período coberto pelo estudo: $\Pi_{t}$ é o valor da produção de leite no Ceará no ano " $\mathrm{t}$ " $(\mathrm{t}=1974,1975, \ldots ., 2019)$, atualizados para 2019, que foi utilizado na pesquisa como proxy da renda bruta; $P_{t}$ é o preço médio de leite recebido pelos produtores cearenses no ano $t$, devidamente corrigido para reais de 2019; $Q_{t}$ é a quantidade, em mil litros, produzida de leite no t-ésimo ano pelos criadores cearenses; $V_{t}$ é o número de vacas em lactação; e $R_{\mathrm{t}}$ é a produtividade diária de leite por vaca em lactação.

Por definição, a quantidade produzida de leite no ano $t\left(Q_{t}\right)$ é igual ao tamanho do rebanho $\left(V_{t}\right)$, multiplicado pela produtividade $\left(R_{t}\right)$ de cada vaca:

$Q_{t}=V_{t} \cdot R_{t}$

O valor da produção $\left(\Pi_{t}\right)$, que pode ser entendido como proxy da receita bruta dos produtores de leite, neste caso, é definido pela Equação 2:

$\Pi_{t}=P_{t} \cdot V_{t} \cdot R_{t}$

Tomando os logaritmos das Equações 1 e 2 e calculando os respectivos valores esperados ou previstos, chegam-se aos resultados esboçados nas Equações 3 e 4.

$$
\begin{aligned}
& E\left[\ln \left(Q_{t}\right)\right]=E\left[\ln \left(V_{t}\right)\right]+E\left[\ln \left(R_{t}\right)\right] \\
& E\left[\ln \left(\Pi_{t}\right)\right]=E\left[\ln \left(P_{t}\right)\right]+E\left[\ln \left(V_{t}\right)\right]+E\left[\ln \left(R_{t}\right)\right]
\end{aligned}
$$

Os ajustamentos buscados na pesquisa visaram encontrar os valores esperados ou previstos mostrados nas Equações 3 e 4.

\subsection{Aferições de estabilidade/instabilidade nas variáveis: objetivo "a"}

Para aferir estabilidade/instabilidade das variáveis estudadas, utilizou-se do coeficiente de variação (CV). Por definição, o CV representa o percentual do desvio-padrão sobre a média de uma variável aleatória. Por essa razão, o CV possibilita fazer comparações entre variáveis de naturezas e aferições distintas (Gomes, 1985; Garcia, 1989; Sorensen, 2000).

Quanto menor o CV, mais estável a distribuição dos valores observados em torno da média. Para usar o CV como medida de aferição do grau de estabilidade ou de instabilidade da distribuição de valores em torno da sua média, necessita-se ter definição acerca de seus valores críticos. Gomes (1985) estabeleceu limites para classificação dos CV em experimentação agrícola. As referências utilizadas neste estudo estão na Tabela 1, assim como as amplitudes em que este estudo enquadra os CV das variáveis endógenas e exógenas associadas à produção de leite no Ceará para atender ao primeiro objetivo (objetivo "a") do estudo. 
Tabela 1. Classificação do CV de acordo com a sua amplitude.

\section{Classificação do CV}

Baixo

Médio

Alto

Muito alto

\section{Amplitude do CV}

$\mathrm{CV}<10 \%$

$10 \% \leq \mathrm{CV}<20 \%$

$20 \% \leq \mathrm{CV}<30 \%$

$\mathrm{CV} \geq 30 \%$

Fonte: Gomes (1985).

\subsection{Projeções das variáveis de decisão na produção de leite: objetivo "b"}

Dada uma variável aleatória $\mathrm{Y}_{\mathrm{t}^{\prime}}$ o seu valor esperado, $E\left(Y_{t}\right)$, será diferente se houver choques de informações causados por situações imprevistas no tempo em que se formaram as expectativas. Isso pode ser escrito de acordo com a Equação 5 a seguir:

$Y_{t}=E\left(Y_{t}\right)+\xi_{t}$

Pode-se reescrever Equação 5 da seguinte forma:

$$
E\left(Y_{t}\right)=Y_{t}-\xi_{t}
$$

Para que seja possível fazer previsões associadas às Equações 5 e 6, é necessário que a série da variável aleatória $Y_{t}$ seja estacionária e o termo $\xi_{t}$ seja ruído branco. Isso significa que a série $\xi_{t}$ precisa ter média zero, variância constante e autocorrelação nula (Box \& Jenkins, 1976; Wooldridge, 2013; Clement, 2014; Camelo et al., 2018; Box et al., 2015).

As formulações propostas por Box \& Jenkins (1976) - ARIMA (Auto-Regressive Integrated Moving Average) - são arcabouços matemáticos que visam captar o comportamento de uma variável aleatória que apresenta valores distribuídos em séries temporais.

Considera-se que a série temporal $Y_{t}$ possa ser representada da seguinte forma:

$Y_{t}=\mu+\sum \psi_{k} \cdot u_{t-k}=\mu+\psi(B) \cdot u_{t}$

em que o filtro linear $\psi$ é definido por:

$\psi(B)=\theta(B) / \phi(B)$

Os termos da Equação 8 são definidos pelos seguintes polinômios:

$\theta(B)=1-\theta_{1} B-\theta_{2} B^{2}-\ldots .-\theta_{q} B^{q} e \phi(B)=1-\phi_{1} B-\phi_{2} B_{2}-\ldots-\phi_{p} B^{p}$

Definindo $\tilde{Y}_{t}=Y_{t}-\mu$, é possível obter a seguinte transformação:

$\phi(B) \tilde{Y}_{t}=\theta(B) u_{t}$

Na Equação 9, $u_{t}$ é um ruído branco geralmente gaussiano. Para tanto, tem que atender às seguintes condições: i) $E\left(u_{t}\right)=0$; ii) $E\left(u_{t}^{2}\right)=\sigma_{u}{ }^{2}<\infty$; e iii) $E\left(u_{t^{\prime}} u_{t+k}\right)=0$, para $k= \pm 1, \pm 2, \ldots$ (Cochrane, 1997). 
De acordo com Box \& Jenkins (1976), a Equação 9 é denominada ARMA(p,q) e pode ser reescrita da seguinte forma:

$\tilde{Y}_{t}=\theta(B) \phi^{-1} \cdot(B) u_{t}$

Os tipos de modelos de Box e Jenkins são:

i) Modelos médias móveis (MA): são aqueles em que $\varphi(B)=1$ e se diz que são $M A(q)$.

ii) Modelos autorregressivos (AR): são aqueles em que $\theta(B)=1$ e se diz que são $A R(p)$. Esses modelos são assim chamados porque $\mathrm{Y}_{\mathrm{t}^{\prime}}$ no instante $\mathrm{t}$, é função dos valores dessa variável em tempos anteriores a t.

iii) Modelos autorregressivos de médias móveis (ARMA): são aqueles que têm uma parte (AR) com uma parte MA e têm a notação $\operatorname{ARMA}(p, q)$.

Antes da aplicação do modelo ARIMA, é necessário avaliar se a série que está sendo analisada é estacionária, isto é, feita observando o gráfico da série e analisando as funções de autocorrelação e autocorrelação parcial (Makridakis et al., 1998). Outra forma de verificação para estacionariedade é estimar o modelo AR em primeira ordem na série original e testar se o coeficiente $\phi$ é menor do que 1.

Um processo estocástico $Y_{t}=\psi(B) u_{t}$ será estacionário se:

$\psi(B)=\Sigma_{k=0}^{\infty} \psi_{k}(B)^{k}$ convergir para $|B|<1$.

Caso a série temporal em estudo não seja estacionária, faz-se necessário transformá-la para se tornar estacionária. Estima-se a função de autocorrelação entre os resíduos. Caso a função de autocorrelação se estabilize com a primeira defasagem, pode-se assegurar que a série passou a ser estacionária. Caso contrário, realizam-se a segunda, terceira ou mais diferenças para buscar a estacionariedade. Em geral, as séries não precisam mais do que três defasagens para se tornarem estacionárias (Morretin \& Toloi, 1987; Kwiatkowski et al., 1992; Makridakis et al., 1998; Li et al., 2019).

\section{Testes de aferição das qualidades dos ajustamentos}

$\mathrm{Na}$ escolha dos modelos estatisticamente apropriados, um dos critérios foi buscar os mais parcimoniosos, na perspectiva de que, quanto menor o número de regressores, melhor o modelo de ajustamento. Além deste, utilizou-se de mais cinco critérios para avaliar as adequações dos modelos ajustados aos objetivos buscados na pesquisa. Foram eles: coeficiente de determinação $\left(\mathrm{R}^{2}\right)$; raiz do erro quadrático médio (RMSE); porcentagem do erro absoluto médio (MAPE); critério bayesiano de Schwarz (SBIC); critério de informação de Akaike (AIC); variância da série $\left(\sigma^{2}\right)$; e estatística Q de Ljung-Box. Todas as estimativas do trabalho foram realizadas usando o software Statistical Package for the Social Sciences (SPSS), versão 20.0 (Makridakis et al., 1998; Wooldridge, 2013; Clement, 2014; Box \& Jenkins, 1976; Greene, 2012; Box et al., 2015; Wang et al., 2018).

\subsection{Estratégias metodológicas para atingir os objetivos "c" e "d"}

Tendo sido ajustados os modelos de previsão de cada uma das variáveis de definição da produção de leite no semiárido do Ceará, efetuaram-se testes para avaliar que variável poderia afetar os choques aleatórios em cada uma das séries ajustadas. 
Neste trabalho, assumiu-se que, no caso da previsão do tamanho do rebanho e da produtividade do leite, os choques $\xi_{t}$ que aparecem nas Equações 5 e 6 foram causados pelas variáveis exógenas: precipitação de chuvas $\left(C_{t}\right)$ e preço médio do leite $\left(P_{t}\right)$. Assim, explicita-se 0 ruído $\xi_{t}$ associado às projeções da produção de leite da forma como mostrado na Equação 12:

$\xi_{t}=f\left(C_{t} ; P_{t}\right)$

Para tanto, define-se a seguinte equação:

$\xi_{t}=\lambda_{0}+\lambda_{1} \ln \left(C_{t}\right)+\lambda_{2} \ln (P t)+v_{t}$

Na Equação 13, os coeficientes $\lambda_{\text {um }}$ e $\lambda_{\text {dois' }}$, sendo estatisticamente diferentes de zero, sinalizam que a precipitação de chuvas no Ceará, para o período analisado, e os preços médios do leite são fatores aleatórios exógenos que afetam os ruídos associados às projeções dos tamanhos e das produtividades desse rebanho no estado. Como as variáveis estão medidas em logaritmos, esses coeficientes se constituem, respectivamente, nas elasticidades que aferem as variações proporcionais nos ruídos, decorrentes da variação de $1 \%$ na precipitação de chuvas e de $1 \%$ nos preços. Na prática, essas elasticidades medem as variações entre as projeções feitas para a variável de decisão que gerou o ruído e o seu valor observado. Quanto maiores forem as magnitudes desses coeficientes em módulo, independentemente do sinal, mais dificuldade será encontrada na formatação de previsões da variável de decisão geradora do ruído. O coeficiente $\lambda_{0}$ é o parâmetro log-linear e $v_{t}$ se constitui no termo aleatório associado a essa equação que, por hipótese, tem média zero, variância constante e não é autorregressivo. Sob essas hipóteses, os parâmetros dessa Equação 13 podem ser estimados usando o método dos mínimos quadrados ordinários (Wooldridge, 2013).

\section{Resultados e discussão}

Começa-se a discussão do trabalho apresentando-se as estatísticas descritivas associadas às variáveis envolvidas na produção de leite no Ceará entre 1974 e 2019.

\subsection{Estatísticas descritivas}

Apresentam-se os valores extremos (mínimos e máximos), médias e coeficientes de variação (CV) da pluviometria anual, valor da produção (em valores de 2019), produção (litros por ano), tamanho do rebanho produtor de leite (número de vacas em lactação), produtividade (litros anuais por vaca) e preço médio do leite em valores de 2019 ( $R \$ /$ litro).

Inicia-se a discussão desses resultados chamando atenção para a grande heterogeneidade associada a praticamente todas as variáveis estudadas. A pluviometria foi bastante instável, como esperado, em se tratando de semiárido, variando de 360,3 milímetros, que foi a grande estiagem ocorrida em 1983, a 1.773,4 milímetros, que se constituiu na maior chuva ocorrida no Ceará no período avaliado, que aconteceu em 1985, dois anos depois da maior estiagem observada na série. A média de pluviosidade no período foi de 788,3 milímetros. O CV de 36,2\% foi classificado como muito alto, de acordo com a métrica estabelecida por Gomes (1985). Essa informação confirma a grande instabilidade da pluviometria cearense entre 1974 e 2019 (Tabela 2).

Além da pluviometria anual, foram considerados como muito altos os $\mathrm{CV}$ estimados para o preço do leite $(C V=51,7 \%)$ e para a produção de leite $(C V=35,8 \%)$. O valor da produção com $C V=21,9 \%$ 
e a produtividade de leite com CV $=20,7 \%$ foram considerados como altos. O tamanho médio anual do rebanho apresentou o menor CV (17,4\%), que foi considerado como médio (Tabela 2).

Das evidências mostradas na Tabela 2, depreende-se que as instabilidades nas pluviometrias observadas no estado do Ceará entre 1947 e 2019 se transferem para as variáveis associadas à produção de leite no mesmo período.

Tabela 2. Estatísticas descritivas associadas às variáveis envolvidas no estudo.

\begin{tabular}{lcccc}
\multicolumn{1}{c}{ Variável } & Mínimo $(\mathbf{m m}) \mathbf{1}$ & Máximo $(\mathbf{m m}) \mathbf{1}$ & Média & CV (\%) \\
Pluviometria & 360,30 & 1773,40 & 788,27 & 36,20 \\
Valor da produção & 429283,90 & 1128470,00 & 661854,17 & 21,87 \\
Produção & 148845,00 & 528138,00 & 308209,67 & 35,81 \\
Rebanho & 304591 & 580949 & 439112 & 17,44 \\
Produtividade & 484,33 & 988,14 & 681,47 & 20,68 \\
Preço médio & 1,23 & 5,01 & 2,55 & 51,66 \\
\hline
\end{tabular}

Fonte: elaborada pelos autores com dados originais da FUNCEME (2020) e IBGE (vários anos).

Desses resultados, observam-se as dificuldades que os criadores de leite no Ceará têm para fazer o planejamento da sua produção, dado o regime pluviométrico do estado. Reunir essas informações para fazer projeções não deve ser tarefa fácil para esses criadores. Isso será observado no tópico seguinte, em que se mostram os resultados encontrados na formatação das projeções associadas às variáveis endógenas de decisão na produção de leite: tamanho do rebanho e produtividade, que está relacionada às tecnologias que os produtores utilizam. Mostra-se também a projeção associada à variável exógena, preço médio do leite, que não é definida sob o domínio desses criadores que são tomadores dessa variável ou ajustadores de quantidades, dados os preços que esperam que aconteçam ao longo do tempo.

\subsection{Formações das projeções}

Os resultados associados ao logaritmo do tamanho do rebanho no ano $\mathrm{t}\left(\mathrm{V}_{\mathrm{t}}\right)$, da produtividade $\left(R_{t}\right)$ e dos preços $\left(P_{t}\right)$ estão apresentados na Tabela 3.

Os ajustamentos para os logaritmos do tamanho do rebanho e para a produtividade foram do tipo ARIMA $(1,0,0)$ : autorregressivos de primeira ordem. Para fazer projeções acerca da variável exógena, logaritmo do preço médio do leite, o melhor ajustamento foi um modelo $\operatorname{ARIMA}(1,0,1)$, como se depreende das evidências mostradas na Tabela 3.

Tabela 3. Estimativa dos parâmetros dos modelos ARMA para as expectativas dos logaritmos do tamanho do rebanho $\left(V_{t}\right)$, produtividade $\left(R_{t}\right)$ e preço médio do leite $\left(P_{t}\right)$.

\begin{tabular}{|c|c|c|c|c|c|c|}
\hline \multirow{2}{*}{ Variável } & \multirow{2}{*}{ Constante } & \multicolumn{2}{|c|}{$\begin{array}{c}\text { Coeficientes } \\
\text { angulares }^{1}\end{array}$} & \multirow{2}{*}{$\begin{array}{c}\text { R2 } \\
\text { Estacionário }\end{array}$} & \multicolumn{2}{|c|}{ Ljung-Box Q } \\
\hline & & $A R(1)$ & MA(1) & & GL & Estatística \\
\hline \multirow[t]{2}{*}{$\ln (\mathrm{Vt})$} & 12,959 & 0,952 & - & 0,835 & 18 & 12,197 \\
\hline & 0 & 0 & & & & $-0,788$ \\
\hline \multirow[t]{2}{*}{$\ln (\mathrm{Rt})$} & 6,525 & 0,98 & - & 0,861 & 18 & 14,717 \\
\hline & 0 & 0 & & & & $-0,616$ \\
\hline \multirow[t]{2}{*}{$\ln (P t)$} & 0,966 & 0,985 & 0,304 & 0,87 & 16 & 17,794 \\
\hline & $-0,206$ & 0 & $-0,06$ & & & $-0,336$ \\
\hline
\end{tabular}

Fonte: elaborada pelos autores com base nos dados da Pesquisa Pecuária Municipal (vários anos). ${ }^{1}$ Valor entre parênteses representa as significâncias das estimativas que foram feitas pelos métodos "Máxima Verossimilhança Exata" e "Cochrane-Orcutt". 


\subsection{Influência das variáveis exógenas sobre os ruídos gerados nas projeções do tamanho do rebanho, produtividade e preço médio do leite}

Os ajustamentos dos impactos dos logaritmos das pluviometrias e dos preços (variáveis exógenas para os produtores de leite) sobre os ruídos associados ao tamanho do rebanho mostraram significância estatística, com erro de, no máximo, 3,6\% (Tabela 4).

Esse resultado confirma a hipótese do trabalho de que as instabilidades pluviométricas e os preços impactaram as previsões do tamanho dos rebanhos por parte dos produtores de leite no Ceará entre 1974 e 2019. Neste caso, observa-se que, em módulo, a instabilidade imprevisível da pluviometria afetava de uma forma bem mais expressiva (elástica) do que a instabilidade dos preços (inelástica) do leite. Os valores estimados, em módulo, foram de, respectivamente, $|1,490|$ e $|0,336|$. Esses resultados confirmam as expectativas feitas na elaboração da pesquisa. Mostram também os impactos mais que proporcionais (elasticidade $=1,49$ ) das variações das precipitações de chuvas sobre os erros de previsão do tamanho do rebanho. Trata-se de um resultado que sinaliza que o planejamento do tamanho do rebanho, que é a fonte geradora da receita do produtor de leite, era fortemente impactado pelas instabilidades pluviométricas no Ceará (Tabela 4).

O sinal negativo associado à elasticidade dos resíduos gerados pela projeção do tamanho do rebanho, impactada pelos preços do leite, sinaliza que os criadores tendiam a compensar as instabilidades de preços com a elevação do tamanho do rebanho, mas de uma forma menos que proporcional, tendo em vista que a elasticidade, neste caso, era negativa e bem menor do que $1(-0,336)$, para evitar que não houvesse queda no valor da produção, que, como se viu na Equação 1, é obtido pela multiplicação da quantidade produzida de leite pelo preço. Isso fica mais evidente nos resultados alcançados nas respostas dos resíduos gerados na previsão da produtividade às oscilações de preços (Tabelas 4 e 5 ).

Tabela 4. Efeitos dos logaritmos das chuvas $\left(C_{t}\right)$ e dos preços médios do leite $\left(P_{t}\right)$ sobre os ruídos extraídos da equação das previsões associadas ao tamanho do rebanho leiteiro no Ceará $\left(V_{t}\right)$ entre 1974 e 2019.

\begin{tabular}{cccc} 
Variável & Coeficiente & Estatística t & Significância \\
$\ln (\mathrm{Ct})$ & 1,490 & 1,822 & 0,000 \\
$\ln (\mathrm{Pt})$ & $-0,336$ & $-2,178$ & 0,036 \\
$\mathrm{R}^{2}$ & 0,998 & & \\
\hline
\end{tabular}

Fonte: elaborada pelos autores com base nos dados da Pesquisa Pecuária Municipal (vários anos).

Na Tabela 5, mostram-se os impactos dos logaritmos das pluviometrias e dos preços sobre os ruídos associados às previsões estimadas de produtividade do leite no Ceará entre $1974 \mathrm{e}$ 2019. As significâncias estatísticas dos coeficientes associados às estimativas foram menores que $1 \%$. Esses resultados são sinalizadores de que essas variáveis exógenas também influenciavam esses ruídos. Neste caso, a imprevisibilidade da instabilidade das chuvas (elasticidade $=0,52$ ) afetava mais essa projeção do que aquela associada aos preços, cuja elasticidade em módulo foi de $|0,441|$. Os resultados também sinalizam que a instabilidade pluviométrica impactou, de forma menos que proporcional e na mesma direção, os erros de previsão da produção diária de leite. Por outro lado, as evidências encontradas também indicam que a instabilidade nos preços do leite também impactou, de forma menos que proporcional (em uma magnitude menor do que a provocada pela instabilidade pluviométrica) e na direção oposta, os erros de previsão associados à produtividade (Tabela 5).

A sinergia entre os resultados mostrados na Tabela 4 e na Tabela 5 sugere que as instabilidades de preços e as projeções dos ruídos associados ao tamanho do rebanho e ao da produtividade das vacas em lactação seguem o mesmo comportamento: com elasticidades negativas. Essas evidências mostram que os produtores de leite planejavam a elevação do número de vacas e 
o incremento da produtividade. Como o valor da produção de leite (proxy da receita bruta) é dado pela multiplicação da produtividade pelo número de vacas em lactação e pelo preço, então um rebanho maior produzindo diariamente mais leite por vaca compensará a instabilidade provocada pela oscilação dos preços.

Tabela 5. Efeitos dos logaritmos das chuvas $\left(C_{t}\right)$ e dos preços médios do leite $\left(P_{t}\right)$ sobre os ruídos extraídos da equação das previsões associadas à produtividade na produção de leite no Ceará $\left(R_{t}\right)$ entre 1974 e 2019.

\begin{tabular}{cccc} 
Variável & Coeficiente & Estatística t & Significância \\
$\ln (\mathrm{Ct})$ & 0,520 & 66,247 & 0,000 \\
$\ln (\mathrm{Pt})$ & $-0,441$ & $-6,583$ & 0,000 \\
$\mathrm{R}^{2}$ & 0,997 & & \\
\hline
\end{tabular}

Fonte: elaborada pelos autores com base nos dados da Pesquisa Pecuária Municipal (vários anos).

\subsection{Comparações dos valores observados com os valores esperados da produção e do valor da produção de leite}

As comparações das produções observadas com as projetadas, bem como dos valores da produção observados com os esperados, foram feitas por meio dos logaritmos naturais dessas variáveis, como se viu na seção de metodologia. Com esses valores que estão apresentados na Figura 1, pode-se citar as evoluções da produção observada e da produção esperada de leite entre 1974 e 2019. Lembrando que o logaritmo da quantidade produzida de leite é dado por:

$\ln \left(Q_{t}\right)=\ln \left(V_{t}\right)+\ln \left(R_{t}\right)$

e que o valor esperado ou projetado do logaritmo da quantidade produzida de leite é dado por: $E\left[\ln \left(Q_{t}\right)\right]=E\left[\ln \left(V_{t}\right)\right]+E\left[\ln \left(R_{t}\right)\right]$

Lembrando também que os valores esperados dos logaritmos dos totais dos rebanhos e das produtividades anuais de leite foram estimados levando em consideração o impacto dos logaritmos das chuvas e dos preços (variáveis exógenas) sobre os ruídos gerados a partir das estimativas dos modelos ARIMA $(1,0,0)$, estimados nesses casos.

Observando-se as trajetórias dos valores observados e esperados ou projetados, depreende-se que os modelos gerados na pesquisa mostraram uma grande aproximação entre os valores observados e esperados, o que pode ser um indicador de robustez dessas estimativas, nesse caso (Figura 1).

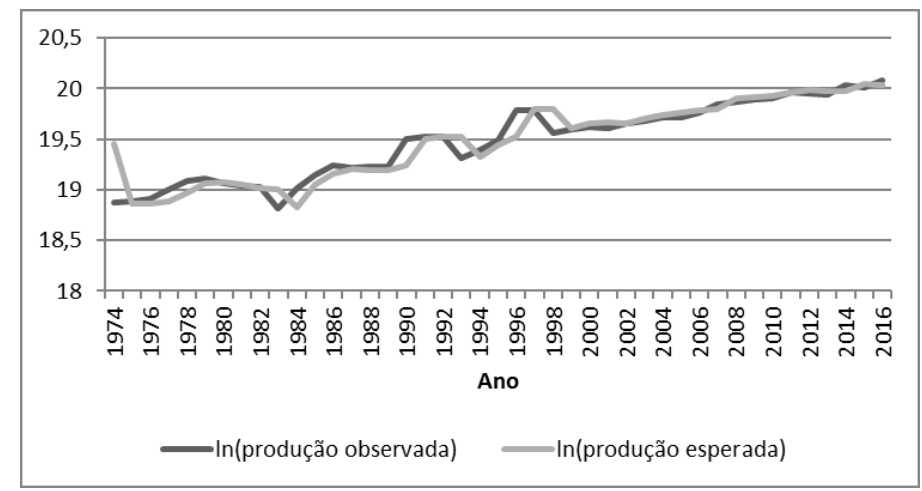

Figura 1. Evolução dos logaritmos dos valores observados e das projeções da produção de leite no Ceará entre 1974 e 2019. Fonte: elaborada pelos autores com base nos dados da Pesquisa Pecuária Municipal (vários anos) e da FUNCEME. 
As comparações dos valores da produção de leite observados e esperados foram estimadas em logaritmos naturais. Ressalta-se que o valor da produção observado de leite $\left(\Pi_{t}\right)$ é dado pela equação:

$\ln \left(\Pi_{t}\right)=\ln \left(V_{t}\right)+\ln \left(R_{t}\right)+\ln \left(P_{t}\right)$

e que o logaritmo do valor da produção esperado, ou projetado, é dado pela equação:

$E\left[\ln \left(\Pi_{t}\right)\right]=E\left[\ln \left(V_{t}\right)\right]+E\left[\ln \left(R_{t}\right)\right]+E\left[\ln \left(P_{t}\right)\right]$

Os valores esperados do logaritmo do tamanho do rebanho $\left(V_{t}\right)$ e da produtividade do rebanho leiteiro $\left(R_{t}\right)$ foram estimados, como se viu, a partir do modelo $\operatorname{ARIMA}(1,0,0)$ e considerando $o$ impacto das variáveis exógenas $\ln \left(P_{t}\right)$ e $\ln \left(C_{t}\right)$ sobre os ruídos gerados nesses modelos.

O valor projetado dos logaritmos dos preços, por sua vez, foi gerado a partir do modelo ARIMA $(1,0,1)$, e os ruídos gerados neste modelo não foram afetados pela variável exógena (logaritmo da pluviometria). Na Figura 2, apresentam-se as trajetórias dos valores observados e esperados da produção de leite no Ceará entre 1974 e 2019, considerando os ajustamentos feitos na pesquisa.

Observa-se que os ajustamentos feitos proporcionaram trajetórias das expectativas dos logaritmos dos valores da produção, praticamente acompanhando a tendência do que aconteceu com os logaritmos dos valores observados. Observa-se também que, a partir de 1990, a maior parte da trajetória dos valores esperados foi mais otimista do que os valores observados (Figura 2).

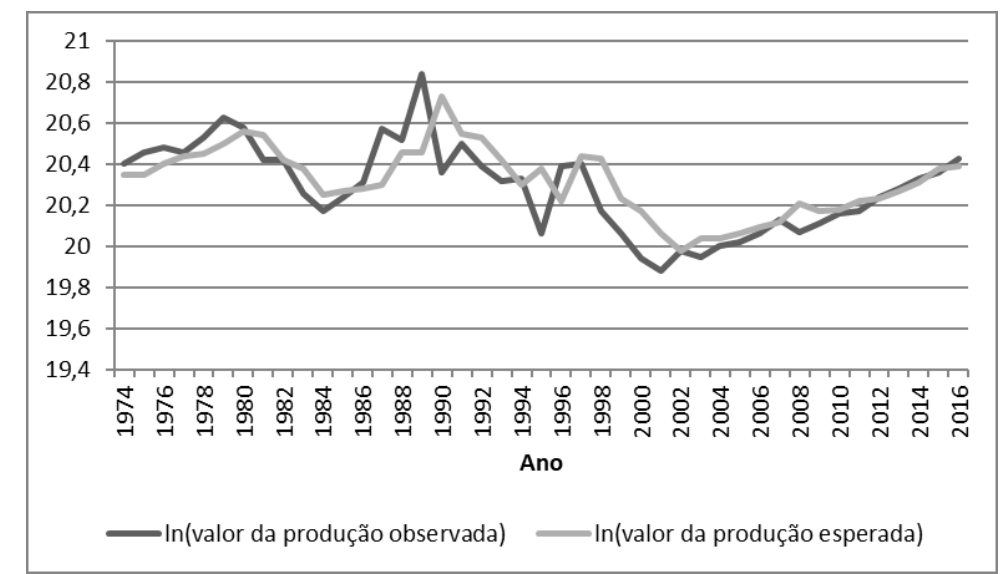

Figura 2. Trajetórias dos valores observados e das projeções dos valores da produção de leite no Ceará entre 1974 e 2019. Fonte: elaborada pelos autores com base nos dados da Pesquisa Pecuária Municipal (vários anos) e da FUNCEME.

A síntese desses resultados é que as variáveis sobre as quais os criadores de leite detêm poder de decisão (tamanho do rebanho) e as expectativas geradas proporcionaram ajustes bastante seguros, de tal sorte que as trajetórias das produções observadas e esperadas "caminharam" bastante próximas ao longo do período estudado. Como os ruídos associados aos valores esperados das variáveis definidoras da produção são afetados pelas variáveis exógenas (precipitação de chuvas e preços), os modelos estimados conseguiram captar essas influências, como se depreende das trajetórias observadas na Figura 2. 
Essas assertivas podem ser estendidas para a trajetória do valor da produção que agrega os preços, além dos tamanhos dos rebanhos e produtividade desse rebanho. Observa-se também que o modelo conseguiu incorporar os valores esperados com bastante rigor, como se infere da Figura 2.

\section{Conclusões}

As evidências apresentadas sugerem que todos os objetivos da pesquisa foram alcançados. A distribuição das chuvas no Ceará entre 1974 e 2019 se mostrou bastante instável, com apenas 12 anos de normalidade, 17 anos com pluviometria considerada como ocorrência de estiagem e 16 anos de períodos chuvosos. O coeficiente de variação associado a essa distribuição pluviométrica, considerado muito alto na escala definida por Gomes (1985), reforça o resultado da instabilidade pluviométrica no Ceará durante o período analisado.

Essa instabilidade se reflete também na heterogeneidade das distribuições das variáveis associadas à produção de leite no Ceará. As expectativas associadas às variáveis endógenas, sobre as quais os produtores têm controle (tamanho do rebanho e produtividade), mostraram sensibilidade às variáveis exógenas, sobre as quais os criadores não têm qualquer poder de decisão: pluviometria, que é um fenômeno natural, e preços do leite, definidos pelo mercado.

Nestes casos, os resultados sinalizam que os criadores, ao planejarem o tamanho do rebanho e a produtividade (produção diária de leite por vaca), reagiam positivamente às instabilidades pluviométricas, aumentando o rebanho e incrementando a produtividade. Em relação às instabilidades dos preços, tanto o tamanho dos rebanhos como a produtividade tinham reação negativa a essas instabilidades. Isso sinaliza que os criadores reagiam a essa instabilidade tentando manter a sua receita bruta, que é obtida pelo produto da produção pela produtividade. Com mais vacas produzindo com maior produtividade, as receitas podiam ser mantidas, mesmo em cenários de queda de preços.

Conclui-se ainda que os modelos gerados foram oriundos de bons ajustamentos, de tal sorte que os valores esperados das produções anuais e dos valores da produção, usados como proxies da renda bruta, aferidos nos respectivos logaritmos, mostraram trajetórias muito próximas daquelas observadas, também aferidas em logaritmos.

A conclusão geral é que a instabilidade na pluviometria observada no Ceará entre 1974 e 2019 exerceu papel importante no comportamento das projeções das variáveis que definem a produção de leite no Ceará sobre as quais os criadores têm algum controle.

\section{Referências}

Box, G. E. P., \& Jenkins, G. M. (1976). Time series analysis forecasting and control. San Francisco: Holden-Day.

Box, G. E., Jenkins, G. M., Reinsel, G. C., \& Ljung, G. M. (2015). Time series analysis forecasting and control. Recuperado em 4 de março de 2021, de http://www.ru.ac.bd/stat/wp-content/ uploads/sites /25/2019/03/504_05_Box_Time-Series-Analysis-Forecasting-and-Control-2015. pdf

Brasil. Ministério da Agricultura, Pecuária e Abastecimento. (2011). Instrução Normativa, $\mathrm{n}^{\circ}$ 62 , de 29 de dezembro de 2011. Regulamenta a produção, identidade, qualidade, coleta e transporte do leite tipo A, leite cru refrigerado e leite pasteurizado. Diário Oficial [da] República Federativa do Brasil, Brasília. 
Brasil. Ministério da Integração Nacional. Conselho Deliberativo da Superintendência do Desenvolvimento do Nordeste - CONDEL/SUDENE. (2017). Resolução n 115, de 23 de novembro de 2017. Diário Oficial [da] República Federativa do Brasil, Brasília. Recuperado em 20 de março de 2021, de https://www.in.gov.br/materia/-/asset_publisher/Kujrw0TZC2Mb/ content/id/739568/do1-2017-12-05-resolucao-n-115-de-23-de-novembro-de-2017-739564

Camelo, H. N., Lucio, P. S., Leal Junior, J. B. V., \& Carvalho, P. C. M. (2018). Modeling Box-Jenkins applied a prediction of wind speed in the regions of the Brazilian northeast for the fins of wind generation. Ciência e Natura, 40, 16. http://dx.doi.org/10.5902/2179460X29785.

Clement, E. P. (2014). Using Normalized Bayesian Information Criterion (Bic) to Improve Box -Jenkins Model Building. American Journal of Mathematics and Statistics, 4(5), 214-221. Recuperado em 10 de março de 2021, de https://www.researchgate.net/publication/297709535_Using_ Normalized_Bayesian

Cochrane, J. H. (1997). Time series for macroeconomic and finances. Graduate School of Business. Recuperado em 12 de junho de 2020, de http://www.bseu.by/russian/faculty5/ stat/docs/4/Cochran,TimeSeries.pdf

Deschênes, O., \& Greenstone, M. (2007). The economic impacts of climate change: evidence from agricultural output and random fluctuations in weather. The American Economic Review, 97(1), 354-385.

Fama, E. (1969). Efficient Capital Markets: a review of theory and empirical work. The Journal of Finance, 25, 383-417.

Folhes, M. T., \& Donald, N. (2007). Previsões tradicionais de tempo e clima no Ceará: o conhecimento popular a serviço da ciência. Sociedade \& Natureza, 19(2), 19-31. http:// dx.doi.org/10.1590/S1982-45132007000200002.

Galvão Júnior, J. G. B., Guilermino, M. M., Medeiros, H. R., Novais, L. P., \& Rangel, A. H. N. (2015). Perfil dos sistemas de produção de leite bovino no Siridó Potiguar. Revista Holos, 2, 130-142.

Garcia, C. H. (1989). Tabelas para classificação do coeficiente de variação (Circular Técnica, No. 171). Piracicaba: IPEF. Recuperado em 2 de março de 2021, de https://www.ipef.br/ publicacoes/ctecnica/nr171.pdf

Gomes, F. P. (1985). Curso de estatística experimental(13. ed.). São Paulo: ESALQ/USP.

Gonçalves, L. C., Borges, I., \& Ferreira, P. D. S. (2015). Alimentação de gado de leite. Belo Horizonte: FEPMVZ-Editora.

Greene, W. H. (2012). Econometric analysis. New York: Pearson.

Instituto Brasileiro de Geografia e Estatística - IBGE. (2006). Censo agropecuário 2006. Recuperado em 10 de agosto de 2021, de https://sidra.ibge.gov.br/tabela/2006

Instituto Brasileiro de Geografia e Estatística - IBGE. (2017). Censo agropecuário 2017. Recuperado em 10 de agosto de 2021, de https://sidra.ibge.gov.br/tabela/2017

Instituto Brasileiro de Geografia e Estatística - IBGE. (2019). Produção pecuária municipal. Rio de Janeiro: IBGE.

Kwiatkowski, D., Phillips, P. C. B., Schmidt, P., \& Shin, Y. (1992). Testing the null hypothesis of stationarity against the alternative of a unit root: how sure are we that economic time series have a unit root. Journal of Econometrics, 54, 159-178.

Lemos, J. J. S. (2020). Vulnerabilidades induzidas no semiárido brasileiro. Desenvolvimento Regional em Debate, 10, 245-268. http://dx.doi.org/10.24302/drd.v10i0.2728. 
Lemos, J. J. S., \& Bezerra, F. N. R. (2019). Interferência da instabilidade pluviométrica na previsão da produção de grãos no semiárido do Ceará. Brazilian Journal of Development, 5(9), 1563215652. http://dx.doi.org/10.34117/bjdv5n9-139.

Li, S., Yang, X., \& Li, R. (2019). Forecasting coal consumption in India by 2030: using linear modified linear (MGM-ARIMA) and linear modified nonlinear (BP-ARIMA) combined models. Sustainability, 11(3), 695. http://dx.doi.org/10.3390/su11030695.

Makridakis, S., Wheelwright, S., \& Hyndman, R. J. (1998). Forecasting methods and applications (3rd ed.). New York: John Wiley \& Sons.

Morretin, P. A., \& Toloi, C. M. C. (1987). Previsão de séries temporais (2a ed.) São Paulo: Atual Editora.

Paiva, E. (2018). Simulações metodológicas para detectar formação de expectativas e tornar a produção de leite sustentável no semiárido cearense (Dissertação de mestrado). Universidade Federal do Ceará, Fortaleza.

Reis Filho, R. J. C., \& Carvalho, A. P. (2009). Plano de desenvolvimento da pecuária leiteira nas áreas irrigáveis do Estado do Ceará. Fortaleza: ADECE.

Silva, F. B. R., Riché, G. R., Tonneau, J. P., Souza Neto, N. C., Brito, L. T. L., Correia, R. C., Cavalcanti, A. C., Silva, F. H. B. B., Silva, A. B., \& Araújo Filho, J. C. (1993). Zoneamento agroecológico do Nordeste: diagnóstico do quadro natural e agrossocioeconômico. Petrolina: Embrapa Semiárido.

Sorensen, A. T. (2000). Equilibrium price dispersion in retail markets for prescription drugs. Journal of Political Economy, 108(4), 833-850. http://dx.doi.org/10.1086/316103.

Thornton, P. K., van de Steeg, J., Notenbaert, A., \& Herrero, M. (2009). The impacts of climate change on livestock and livestock systems in developing countries: a review of what we know and what we need to know. Agricultural Systems, 101(3), 113-127. http://dx.doi. org/10.1016/j.agsy.2009.05.002.

Wang, Q., Li, S., Li, R., \& Ma, M. (2018). Forecasting U.S. shale gas monthly production using a hybrid ARIMA and metabolic nonlinear grey model. Energy, 160, 378-387. http://dx.doi. org/10.1016/j.energy.2018.07.047.

Wooldridge, J. M. (2013). Introductory econometrics: a modern approach (5th ed). Mason: South Western Educational Publishing.

Ximenes, L. F. (2013). Efeitos da ocorrência de secas sobre indicadores agropecuários do Estado do Ceará. Informe Rural, 7(1), 1-14.

Ximenes, L. J. F. (2009). Predição do Lucro por mudança unitária das características produtivas e reprodutivas de bovinos leiteiros no semiárido do Nordeste (Tese de doutorado). Universidade Federal do Ceará, Fortaleza. 